http://jmscr.igmpublication.org/home/ ISSN (e)-2347-176x ISSN (p) 2455-0450 crossref DOI: https://dx.doi.org/10.18535/jmscr/v7i9.53

\author{
Dournal Of Medical Science And Clinical Research \\ IGM Publication \\ An Official Publication of IGM Publication
}

\title{
Effect of Physical Activity on PEFR in Apparently Normal \& Healthy Subjects, a cross sectional analytical study
}

\author{
Authors \\ Dr Manohar Lal Bhandari ${ }^{1}$, Dr Priyanka Verma ${ }^{2 *}$, Dr R. K Choudhary ${ }^{3}$, \\ Dr Sachin Kuchya ${ }^{4}$ \\ ${ }^{1}$ Associate Professor in Physiology, Department of Physiology MGMMC Indore \\ ${ }^{2,3} \mathrm{PG}$ 2nd year, Department of Physiology MGMMC Indore \\ ${ }^{4}$ Professor in Pharmacology, Department of Pharmacology, NSCB MC Jabalpur \\ *Corresponding Author \\ Dr Priyanka Verma
}

\section{Introduction}

In diagnosis and treatment of respiratory diseases, the assessment of lung functions is of considerable importance $^{(1)}$. The Peak Expiratory flow Rate (PEFR) is a simple, reproducible \& easily affordable test of lung function. Standard or Reference PEFR, hence forth called as Reference PEFR values for an individual subject depends upon his / her gender, height, weight, ethnicity \& smoking status ${ }^{(2)}$, are obtained with the help of specific formulae's or with the help of nomograms.

An Individual subject has a PEFR, determined with the help of peak flow meter, the Cipla Breathometer $^{(3)}$ being the most studied one. The PEFR of an individual subject varies with the reference value. A higher deviation is often seen in those with respiratory illness, which is used for diagnosis or treatment of Respiratory Illness. The said deviation is also seen in subjects without history of any Respiratory or related Illness.

This cross sectional study was conducted to measure the PEFR values and an attempt has been made to find out the correlation between PEFR values and dynamic anthropometric parameter such as Chest expansion \& Physical activity.

\section{Material \& Method}

This study was conducted at MGM Medical College, Indore \& Study participants were Undergraduate students pursuing MBBS Ist year and Ist year BPT course. These students were taught, Respiratory physiology and its assessment during their formal training sessions in the department of Physiology. They were invited to be part of this study \& asked to complete a Respiratory health and demographic questionnaire. Those students who duly completed $\&$ submitted the questionnaire were offered Informed consent form. Those who provided the informed consent were then included in the study. (Figure 1)

Following their inclusion in the study, the vitals like height, weight, chest measurements, Peripheral Oxygen saturation (SpO2), Hemoglobin, Pulse Rate and Blood pressure, PEFR were recorded. The Weight, Height, Chest measurements, Peripheral Oxygen saturation 
(SpO2), Hemoglobin, Pulse Rate and Blood pressure were assessed with the help of standard and well validated instruments. The PEFR values were measured using the Breathometer ${ }^{\mathrm{TM}}$ (Cipla Ltd., India) with EU scale. The highest of the three readings were taken as the test value ${ }^{(4)}$.
Reference values of PEFR, for each subject were calculated with the help of equations derived and validated in a previous study. The formulae for determining Reference PEFR were, for Females: $\mathrm{PEF}=-1.454$ (Age) +2.368 (Height) $\&$ for Males: $\mathrm{PEF}=-1.807($ Age $)+3.206$ (Height) ${ }^{(3)}$

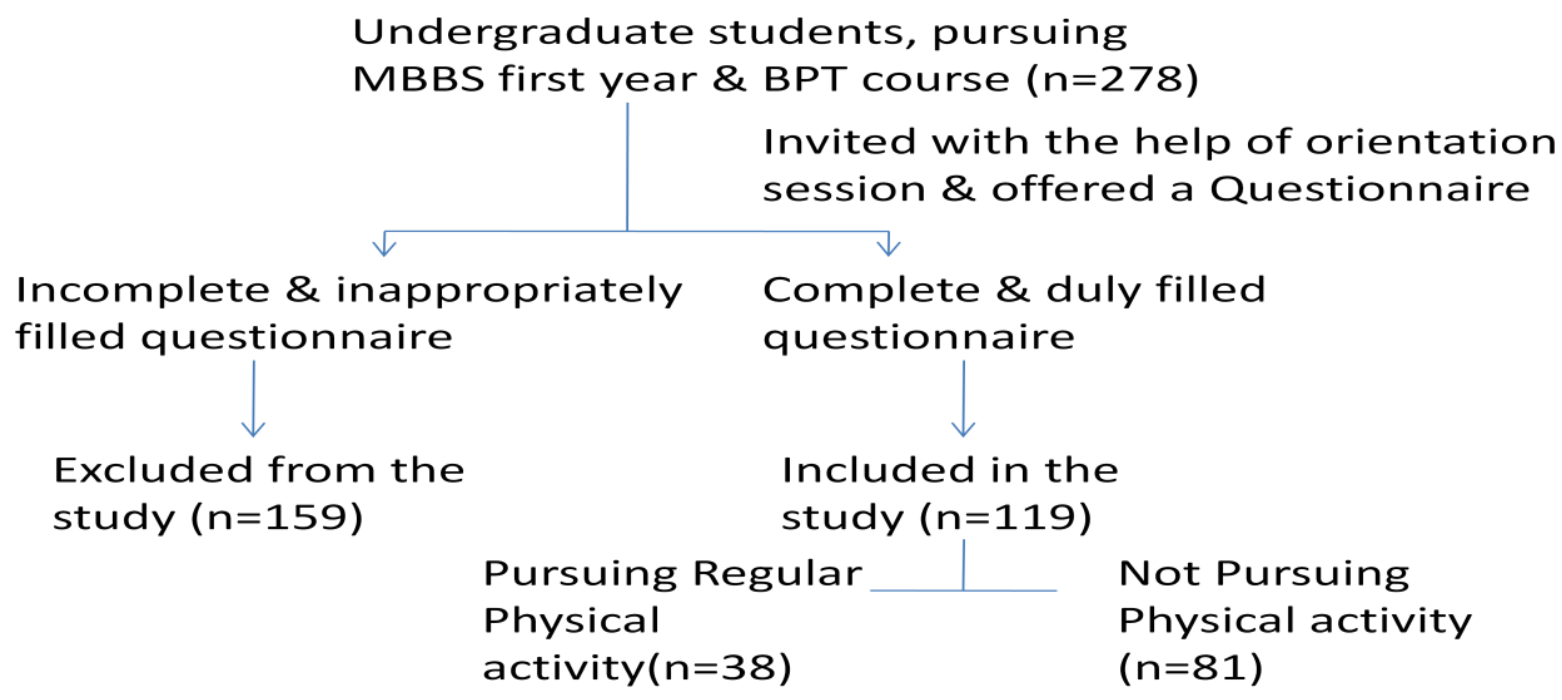

The data was tabulated on an Excel sheet, analyzed with the help of suitable filters. The data sets thus obtained, were analyzed with the help of students $\mathrm{T}$ test Unpaired assuming unequal variances. $^{(5)}$

\section{Result}

In all 278 students were invited and as per study flow, 119 were included in the study. There were 78 female (age, $19.6 \pm 0.26)$ and 41 male $(19.2 \pm$ 0.32 ) subjects in the study. All the subjects included in the study, were healthy \& nonsmokers. None of them reported suffering from any Respiratory disorder. They had peripheral Oxygen saturation (SpO2), Hemoglobin, Pulse Rate and Blood pressure, within Normal range.

The PEFR values \& related data were first grouped separately for males \& females. Then among males \& females, they were further

grouped on the basis of Regular Physical activity (Yes / No); On type of physical activity Isotonic (Yoga, Pranayam, Aerobics, Dancing, walking and stretching etc) \& Isometric (weight training, pushups, gymming etc); Chest expansion $(<5 \mathrm{~cm}$ vs $\geq 5 \mathrm{~cm}$ ) (10); Duration of activity $(<150$ minutes per week vs $\geq 150$ minutes per week) (79), as detailed in tables below.

The groups once made were matched for variables like age distribution, they matched each other $(p<0.05)$. The PEFR values were then matched with reference values obtained for each individual subject and difference ( $\triangle$ PEFR) was calculated. The mean $\triangle$ PEFR for each group was calculated and compared against comparable group. The $\mathrm{F}$ values (ratio of variance within each group) were calculated and appropriate test of significance was chosen. The Students t-test unpaired, for unequal variances were applied.

Table I_Illustrating the Effect of Physical activity on PEFR in Females

\begin{tabular}{|l|r|r|c|c|}
\hline Regular Physical activity & $\begin{array}{c}\text { No. of } \\
\text { Subjects }\end{array}$ & $\begin{array}{c}\text { Mean } \\
\Delta \text { PEFR }\end{array}$ & $\begin{array}{c}\mathrm{p} \\
\text { value }\end{array}$ & Significance \\
\hline Yes & $\mathbf{1 9}$ & $\mathbf{- 3 1 . 4 8}$ & $\mathbf{0 . 1 5}$ & $\begin{array}{c}\text { Statistically } \\
\text { Insignificant }\end{array}$ \\
\hline None & $\mathbf{5 9}$ & $\mathbf{- 5 6 . 7 5}$ & &
\end{tabular}




\begin{tabular}{|l|c|c|c|c|}
\hline Type of Physical activity & $\begin{array}{c}\text { No. of } \\
\text { Subjects }\end{array}$ & $\begin{array}{c}\text { Mean } \\
\Delta \text { PEFR }\end{array}$ & $\begin{array}{c}\mathrm{p} \\
\text { value }\end{array}$ & Significance \\
\hline Isotonic ( Aerobic) & $\mathbf{1 4}$ & $\mathbf{- 3 7 . 2 7}$ & $\mathbf{0 . 3 3}$ & $\begin{array}{c}\text { Statistically } \\
\text { Insignificant }\end{array}$ \\
\hline Isometric (Gymming) & $\mathbf{5}$ & $\mathbf{- 1 5 . 2 7}$ & & \multicolumn{2}{|r}{} \\
\hline
\end{tabular}

\begin{tabular}{|c|c|c|c|c|}
\hline $\begin{array}{l}\text { Type of Physical activity \& } \\
\text { Chest expansion }\end{array}$ & $\begin{array}{l}\text { No. of } \\
\text { Subjects }\end{array}$ & $\begin{array}{l}\text { Mean } \\
\triangle \text { PEFR }\end{array}$ & $\begin{array}{c}\mathrm{p} \\
\text { value }\end{array}$ & Significance \\
\hline $\begin{array}{l}\text { Isotonic \& Chest Expansion } \\
(<5 \mathrm{~cm})\end{array}$ & 8 & -12.26 & \multirow[t]{2}{*}{0.16} & \multirow[t]{2}{*}{$\begin{array}{l}\text { Statistically } \\
\text { Insignificant }\end{array}$} \\
\hline $\begin{array}{l}\text { Isotonic \& Chest Expansion } \\
(\geq 5 \mathrm{~cm})\end{array}$ & 6 & -70.63 & & \\
\hline $\begin{array}{l}\text { Isometric \& Chest Expansion } \\
(<5 \mathrm{~cm})\end{array}$ & 3 & -20.28 & \multirow[t]{2}{*}{0.45} & \multirow[t]{2}{*}{$\begin{array}{c}\text { Statistically } \\
\text { Insignificant }\end{array}$} \\
\hline $\begin{array}{l}\text { Isometric \& Chest Expansion } \\
(\geq 5 \mathrm{~cm})\end{array}$ & 2 & -7.75 & & \\
\hline
\end{tabular}

\begin{tabular}{|c|c|c|c|c|}
\hline $\begin{array}{l}\text { Type \& Duration of Physical } \\
\text { activity }\end{array}$ & $\begin{array}{l}\text { No. of } \\
\text { Subjects }\end{array}$ & $\begin{array}{c}\text { Mean } \\
\triangle \text { PEFR }\end{array}$ & $\begin{array}{c}\mathrm{p} \\
\text { value }\end{array}$ & Significance \\
\hline $\begin{array}{l}\text { Isotonic \& Duration }<150 \\
\mathrm{~min} / \text { week }\end{array}$ & 5 & 25.28 & 0.03 & $\begin{array}{c}\text { Statistically } \\
\text { significant }\end{array}$ \\
\hline $\begin{array}{l}\text { Isotonic \& Duration } \geq 150 \\
\mathrm{~min} / \text { week }\end{array}$ & 9 & -72 & & \\
\hline $\begin{array}{l}\text { Isometric \& Duration }<150 \\
\mathrm{~min} / \text { week }\end{array}$ & 1 & 77 & \multirow{2}{*}{\multicolumn{2}{|c|}{$\begin{array}{c}\text { Statistics \& Relevant Tests } \\
\text { can't be applied }\end{array}$}} \\
\hline $\begin{array}{l}\text { Isometric \& Duration } \geq 150 \\
\mathrm{~min} / \text { week }\end{array}$ & 4 & -38.58 & & \\
\hline
\end{tabular}

Table II Illustrating the Effect of Physical activity on PEFR in Males

\begin{tabular}{|l|c|c|c|c|}
\hline Regular Physical activity & $\begin{array}{c}\text { No. of } \\
\text { Subjects }\end{array}$ & $\begin{array}{c}\text { Mean } \\
\Delta \text { PEFR }\end{array}$ & p value & Significance \\
\hline Yes & $\mathbf{1 9}$ & $\mathbf{- 9 4 . 1 7}$ & $\mathbf{0 . 1 9}$ & $\begin{array}{c}\text { Statistically } \\
\text { Insignificant }\end{array}$ \\
\hline None & $\mathbf{2 2}$ & $\mathbf{- 7 6 . 8 5}$ & & \\
\hline
\end{tabular}

\begin{tabular}{|l|c|c|c|c|}
\hline Type of Physical activity & $\begin{array}{c}\text { No. of } \\
\text { Subjects }\end{array}$ & $\begin{array}{c}\text { Mean } \\
\Delta \text { PEFR }\end{array}$ & p value & Significance \\
\cline { 1 - 3 } Isotonic (Aerobic) & $\mathbf{1 0}$ & $\mathbf{- 7 1 . 7 8}$ & $\mathbf{0 . 0 3}$ & $\begin{array}{c}\text { Statistically } \\
\text { Significant }\end{array}$ \\
\hline Isometric (Gymming) & $\mathbf{9}$ & $\mathbf{- 1 1 9}$ & & \\
\hline
\end{tabular}

\begin{tabular}{|c|c|c|c|c|}
\hline $\begin{array}{l}\text { Type of Physical activity } \\
\& \text { Chest expansion }\end{array}$ & $\begin{array}{l}\text { No. of } \\
\text { Subjects }\end{array}$ & $\begin{array}{c}\text { Mean } \\
\triangle \text { PEFR }\end{array}$ & $\mathrm{p}$ value & Significance \\
\hline 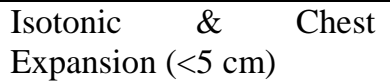 & 5 & -53.35 & \multirow[t]{2}{*}{0.08} & \multirow[t]{2}{*}{$\begin{array}{l}\text { Statistically } \\
\text { Insignificant }\end{array}$} \\
\hline $\begin{array}{lcc}\text { Isotonic } \quad \& & \text { Chest } \\
\text { Expansion }(\geq 5 \mathrm{~cm}) & \end{array}$ & 5 & -90.22 & & \\
\hline $\begin{array}{l}\text { Isometric \& } \quad \text { Chest } \\
\text { Expansion }(<5 \mathrm{~cm})\end{array}$ & 3 & -90.32 & \multirow[t]{2}{*}{0.19} & \multirow[t]{2}{*}{$\begin{array}{l}\text { Statistically } \\
\text { Insignificant }\end{array}$} \\
\hline $\begin{array}{lcl}\text { Isometric \& } & \text { Chest } \\
\text { Expansion }(\geq 5 \mathrm{~cm}) & \\
\end{array}$ & 2 & -127.245 & & \\
\hline
\end{tabular}




\begin{tabular}{|c|c|c|c|c|}
\hline $\begin{array}{l}\text { Type \& Duration of } \\
\text { Physical activity }\end{array}$ & $\begin{array}{l}\text { No. of } \\
\text { Subjects }\end{array}$ & $\begin{array}{c}\text { Mean } \\
\Delta \text { PEFR }\end{array}$ & $\mathrm{p}$ value & Significance \\
\hline $\begin{array}{l}\text { Isotonic \& Duration }<150 \\
\mathrm{~min} / \text { week }\end{array}$ & 3 & -86.72 & 0.03 & $\begin{array}{l}\text { Statistically } \\
\text { significant }\end{array}$ \\
\hline $\begin{array}{l}\text { Isotonic \& Duration } \geq 150 \\
\mathrm{~min} / \text { week }\end{array}$ & 7 & -65.38 & & \\
\hline $\begin{array}{l}\text { Isometric \& Duration } \\
<150 \mathrm{~min} / \text { week }\end{array}$ & $\mathbf{0}$ & $\mathbf{0}$ & \multirow{2}{*}{\multicolumn{2}{|c|}{$\begin{array}{c}\text { Statistics \& Relevant Test } \\
\text { can't be applied }\end{array}$}} \\
\hline $\begin{array}{l}\text { Isometric \& Duration } \\
\geq 150 \mathrm{~min} / \text { week }\end{array}$ & 9 & -119 & & \\
\hline
\end{tabular}

\section{Analysis/Result}

Among the females, those who pursued Regular Physical activity $(\mathrm{n}=19)$ had a lower $\triangle$ PEFR (44\%) as compared to those who didn't $(n=59)$, but it was not significant statistically $(\mathrm{p}=0.15$, $>0.05$ ). Table 1

Among the female subjects, who pursued Regular physical activity, those who pursued Isotonic exercises $(n=14)$ had a higher $\triangle$ PEFR $(144 \%)$ as compared to those who did isometric exercise $(\mathrm{n}=5)$, but this was not significant statistically $(\mathrm{p}=0.33,>0.05)$. Table 2

The differences among those who pursued Isotonic or Isometric exercise \& who had a chest expansion $(<5 \mathrm{~cm}$ or $\geq 5 \mathrm{~cm})$ also were not significant statistically. Table 3

Those who pursued Isotonic ( $<150 \mathrm{~min} /$ week ) $(n=5)$, had PEFR values which were higher as compared to their reference values, while those with Isotonic exercise ( $\geq 150 \mathrm{~min} /$ week) ( $\mathrm{n}=9)$ had lower PEFR as compared to their reference values. The difference is statistically significant. $(\mathrm{p}=0.03,<0.05)$ Table 4.

Among the male subjects, those who pursued Regular Physical activity $(n=19)$ had a Higher $\triangle$ PEFR (122\%), as compared to those who didn't $(\mathrm{n}=22)$, and it was not significant statistically $(\mathrm{p}=0.19,>0.05)$. Table 5 those who pursued Isotonic exercises $(n=10)$ had a lower $\triangle$ PEFR $(40 \%)$ as compared to those who did isometric exercise \& this difference was statistically significant $(\mathrm{p}=0.03,<0.05)$. Table 6

The differences among the male subjects, those who pursued Isotonic or Isometric exercise \& who had a chest expansion $(<5 \mathrm{~cm}$ or $\geq 5 \mathrm{~cm})$ also were not significant statistically. Table 7
Those who pursued Isotonic exercise ( $<150 \mathrm{~min}$ /week), had a higher $\triangle$ PEFR (132\%) as compared to those who pursued Isotonic exercise $(\geq 150 \mathrm{~min}$ / week) \& the difference is statistically significant too $(\mathrm{p}=0.03,<0.05)$ Table 8

\section{Discussion}

The PEFR of subjects varied with that of Reference values for each subject. There was a greater deviation $\triangle$ PEFR among males $(n=41)(-$ $84 \pm 20.2)$ in comparison to females $(n=78)(-$ $50 \pm 16.5)$.

It was statistically significant in females who pursued Isotonic exercise, for duration $<150 \mathrm{~min}$ per week $\&$ in males who pursued Isotonic exercise, Irrespective of duration of activity. They had lower $\triangle$ PEFR values in comparison to subjects who followed either Isometric exercise or none.

In males, those who did Isotonic exercise for duration $\geq 150$ min per week had higher PEFR values as compared to those who pursued isotonic exercise for $<150$ min per week while the reverse was found true in females. This was a contradiction discovered in the study $\&$ should be taken up for validation with the help of a larger study.

The findings of the study agree with the findings of the previous study by Karthik PS ${ }^{(6)}$ et al.

\section{Recommendation}

The study could have been better with a greater participation $(42 \%$ of the Invited subjects, satisfied the Inclusion criteria), a greater proportion of individuals practicing regular physical activity (only around 24\% \& $46 \%$ of subjects pursued regular physical activity, 
amongst females \& males resp.) \& Similar type of activity for a longer duration of time. The participants practiced Isotonic exercises like, Yoga, Pranayam, Aerobics, Dancing, walking and stretching and that too in various proportions. Very few of participants who did pursue the Regular physical activity, did it for $\geq 150$ mins per week.

\section{Conclusion}

The subjects who pursued regular physical activity in the form of Isotonic exercises had PEFR values closer to the Reference values for their Gender, height, Weight. However this benefit needs better Quantification \& validation with a larger \& more robust study.

\section{References}

1. Dhungel KU1, Parthasarathy D, Dipali S. Peak expiratory flow rate of Nepalese children and young adults. Kathmandu Univ Med J (KUMJ). 2008 JulSep;6(23):346-54.

2. Ebomoyi MI1, Iyawe VIVariations of peak expiratory flow rate with anthropometric determinants in a population of healthy adult nigerians. Niger J Physiol Sci. 2005 Jun-Dec;20(1-2):85-9.

3. Kodgule R R, Singh V, Dhar R, Saicharan B G, Madas S J, Gogtay J A, Salvi S S, Koul P A. Reference values for peak expiratory flow in Indian adult population using a European Union scale peak flow meter. J Postgrad Med 2014;60:123-9

4. Islam MN1, Islam MN, Hoque MA, Latif SA, Mollah AH, Hossain MA, Husain MF, Rahman MA, Akhtaruzzaman M, Khan AH, Paul BK, Kundu GK. Peak expiratory flow rate of normal school going children in Mymensingh municipality. Mymensingh Med J. 2013 Jul;22(3):438-43.
5. Gagnier JJ1, Morgenstern $\mathrm{H}$. Misconceptions, Misuses, and Misinterpretations of $\mathrm{P}$ Values and Significance Testing. J Bone Joint Surg Am. 2017 Sep 20;99(18):1598-1603. doi: 10.2106/JBJS.16.01314

6. Karthik PS1, Chandrasekhar M2, Ambareesha K3, Nikhil C4. Effect of pranayama and suryanamaskar on pulmonary functions in medical students. $\mathbf{J}$ Clin Diagn Res. 2014 Dec;8(12):BC04-6. doi: $\quad 10.7860 / J C D R / 2014 / 10281.5344$. Epub 2014 Dec 5.

7. Physical Activity Guidelines Advisory Committee (PAGAC). Physical Activity Guidelines Advisory Committee Report, 2008. Washington, DC, US Department of Health and Human Services, 2008.

8. Bauman A, Lewicka M, Schöppe S. The Health Benefits of Physical Activity in Developing Countries. Geneva, World Health Organization, 2005.

9. Warburton D et al. A systematic review of the evidence for Canada's Physical Activity Guidelines for Adults. International Journal of Behavioural Nutrition and Physical Activity, 2009 [under review for publication].

10. Rajani S. Pagare, Ratnaprabha B. Pedhambkar. ASSESSMENT OF REFERENCE VALUES OF CHEST EXPANSION AMONG HEALTHY ADULTS IN PUNE, INDIA. Int $\mathbf{J}$ Physiother Res 2017;5(1):1819-1823. 